\title{
Eliminating HIV transmission in New South Wales: the critical role of testing
}

\section{The "ending HIV" movement calls for new and innovative approaches to testing}

n Australia, about 1000 new HIV diagnoses are notified annually. In 2012, after 10 years of relative stability, the number of HIV notifications in New South Wales sharply increased. Most other states and territories have experienced a similar trend. There were 409 new notifications of HIV reported in NSW in 2012; a 24\% increase compared with 2011. ${ }^{1}$ In 2013, there were 357 notifications; a fall of 13\% from 2012 but still higher than any year since 2007. Men who have sex with men (MSM) accounted for $78 \%$ of all notifications. $^{2}$

In the 30 years since the first Australian HIV case was recorded in Sydney, approaches to HIV diagnosis, treatment and prevention have evolved in response to emerging scientific evidence. Thanks to the availability of effective antiretroviral treatment, receiving a diagnosis of HIV in Australia is no longer the death sentence it once was. Instead, living with HIV today carries with it the lifelong health and social consequences of a unique chronic disease.

\section{A new era}

In 2011, a large clinical trial demonstrated that antiretroviral treatment not only effectively treats HIV but also prevents HIV transmission within serodiscordant heterosexual couples. ${ }^{3}$ The results were heralded as the scientific breakthrough of $2011^{4}$ and revolutionised HIV prevention by placing treatment-based approaches alongside traditional behavioural interventions. However, the study was not able to assess HIV transmission between MSM owing to insufficient numbers.

In 2014, interim results were released from the second large study to look at the impact of antiretroviral treatment on HIV transmission, ${ }^{5}$ which included a significant cohort of serodiscordant male couples. The results show that treatment also prevents HIV transmission between MSM, with a maximum $1 \%$ per year chance of transmission from any anal sex from someone on successful HIV treatment. Final results from the study will be available in 2017.

The concept of treatment as prevention is not unique to HIV, having long been the keystone in the control of communicable diseases such as tuberculosis and syphilis. However, HIV poses particular challenges to the traditional model of communicable disease control, because the phase of greatest infectiousness is usually soon after

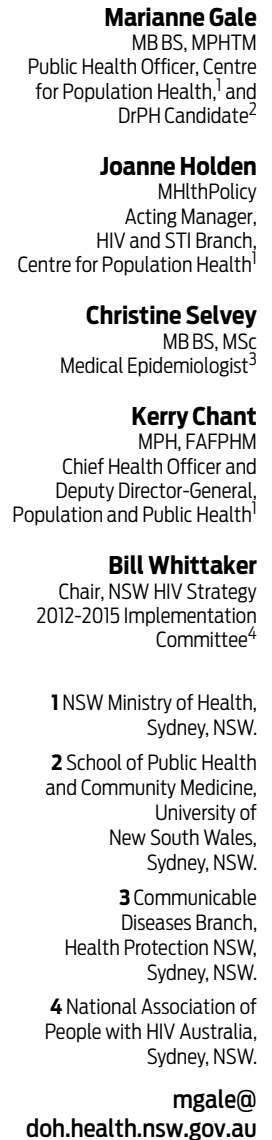

doi: $10.5694 /$ mjal4.00490

Online first: 25/08/14 infection and is often asymptomatic, contact tracing is frequently complex, and treatment is not yet a cure and therefore must be lifelong.

In this new era, where antiretroviral treatment has not only redefined HIV as a chronic disease but is also a powerful addition to the HIV prevention armamentarium, the elimination of HIV transmission can for the first time be considered a potentially achievable goal. In December 2012, the NSW Minister for Health launched the NSW HIV Strategy 2012$2015,{ }^{6}$ which has the overarching goal of working towards the elimination of HIV transmission by 2020. The strategy sets treatment and prevention targets for NSW that align with those defined in the landmark 2011 United Nations political declaration on HIV and AIDS, ${ }^{7}$ to which Australia committed.

\section{Test more, test often}

In this new scientific and political framework, successfully implementing an HIV testing strategy that reduces the pool of undiagnosed HIV infection, detects new infections early and facilitates linkage to care is a key component of HIV prevention. ${ }^{8}$ Modelling of the Australian HIV epidemic in MSM suggests that transmission from people with undiagnosed infection could account for more than $30 \%$ of all new infections. ${ }^{9}$

Free HIV testing targeting the high-risk populations of MSM, injecting drug users and sex workers has long been accessible in clinical settings in NSW. Despite the relative ease of access to clinical services, there remain at-risk individuals who do not engage with the health system and are therefore not tested, or who do engage but are not tested at all or frequently enough. This is especially relevant for MSM, for whom testing up to four times a year may be recommended, depending on individual factors. ${ }^{10}$ Achieving substantial increases in testing rates and changing the pattern of testing among segments of the population of MSM in NSW is essential if the goal of the HIV strategy is to be achieved.

Getting high-risk populations to test frequently can be complex, and evidence for effective approaches is lacking. A qualitative study of MSM in NSW found that testing more frequently would be acceptable if more convenient testing and rapid result turnaround were made available. ${ }^{11}$ Developing new service models to make HIV testing easier, quicker and more accessible is a critical step towards achieving the high coverage and frequency of HIV testing needed. 
A diversity of testing options is essential, recognising that the willingness and ability of at-risk people to test for HIV on a regular basis will be influenced by a range of factors including time constraints, geographical location and perception of risk. Different testing options may not only suit different individuals but also each individual differently depending on the occasion. For example, a gay man may find it most convenient to see his general practitioner once a year for a complete sexual health check including HIV testing, and then visit a community-based site every 3 months for HIV screening with a rapid test. Creating a variety of easy testing options is the task at hand for NSW Health and its health sector, community and academic partners.

\section{Rapid HIV testing}

Since October 2011, rapid HIV testing has become increasingly available in NSW via trials, demonstration projects and routine practice, and it is currently available at more than 19 sites, including three community sites. A range of rapid tests are on the market and allow results to be given to the patient within 30 minutes. The Therapeutic Goods Administration (TGA) has approved one of these tests, the Determine HIV-1/2 Ag/Ab Combo (Alere), for point-of-care use in Australia, and other tests are under review. The use of rapid tests to screen for HIV in novel settings, in conjunction with conventional testing in clinical settings, has the potential to significantly increase testing across the state.

To mark World AIDS Day 2013, NSW Health launched the first ever pop-up HIV testing site in Australia. A caravan, marked with NSW Health and ACON (AIDS Council of NSW) branding, provided a private and confidential space on Oxford Street, Darlinghurst, where clients could drop in for a test (Box). The 5-day site attracted media coverage that reached an estimated audience greater than 4 million people. Over 500 people engaged with the site and 195 opted to have a test, almost all of whom were MSM. No new HIV diagnoses were made. Feedback from clients showed that the model was highly valued (unpublished data). Since that initial experience, further pop-up sites have been established in NSW at the Tropical Fruits New Year's Eve Festival in Lismore and on Oxford Street during the 2014 Sydney Gay and Lesbian Mardi Gras.

NSW local sexual health and laboratory services oversee pop-up sites to ensure compliance with TGA requirements for point-of-care HIV testing. For all rapid-testing sites, including pop-ups, the local service's standard operating procedures ensure that any person with a reactive rapid test is offered prompt laboratory-based confirmatory testing and linkage to comprehensive clinical care and psychosocial support including early assessment for treatment initiation. If a person with a confirmed diagnosis has a regular GP, the sexual health service can inform that GP if

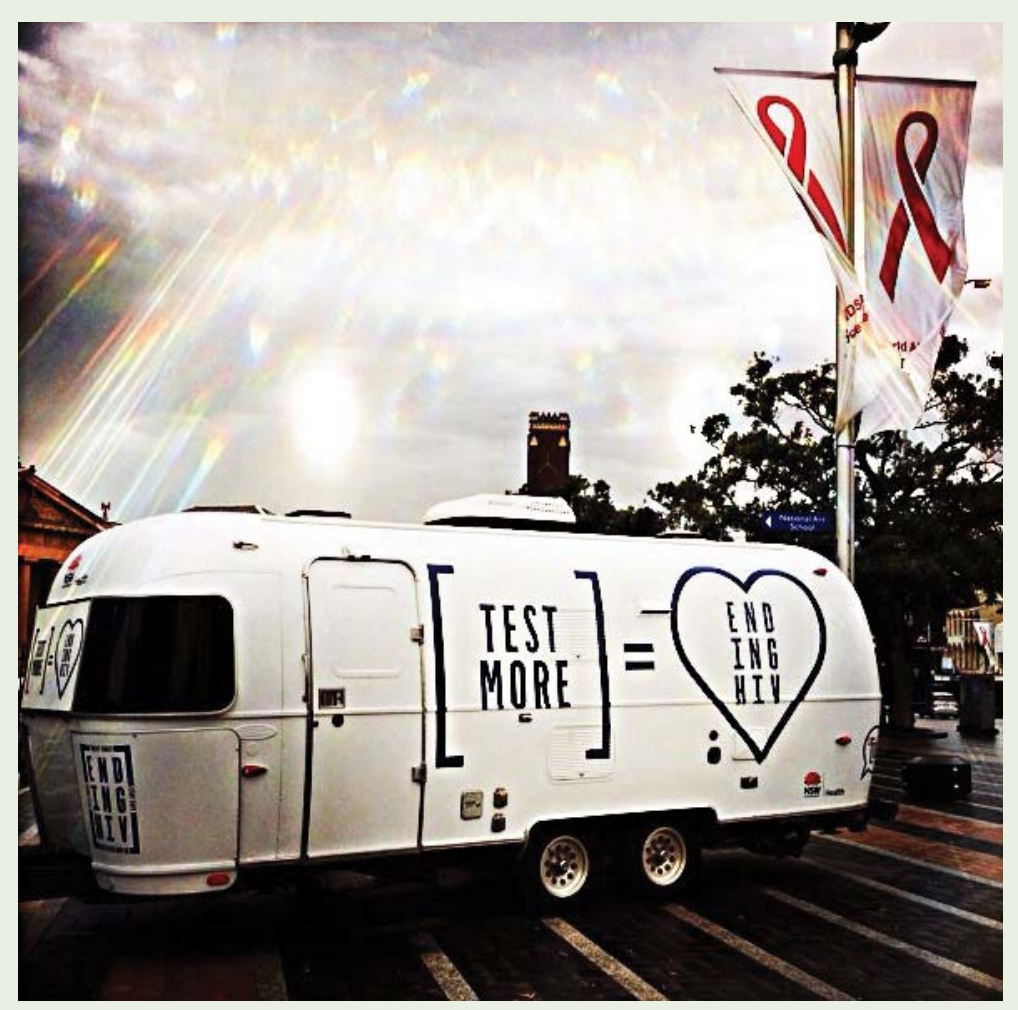

the patient so wishes. Newly diagnosed patients can choose their preferred arrangements for ongoing HIV management. If the regular GP is not an accredited HIV section 100 prescriber, a shared-care model may be adopted.

Additionally, the NSW HIV Support Program helps diagnosing doctors ensure that all people who are newly diagnosed with HIV have timely access to appropriate clinical management, psychosocial support, counselling on HIV treatment and preventing transmission, assistance with tracing partners at risk, and linkage to relevant specialist and community services. ${ }^{12}$

Getting highrisk populations to test frequently can be complex

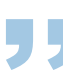

Although making HIV testing facilities visible in public locations may be confronting for some, visibility is important in encouraging open conversation about HIV and normalising testing, especially in areas with high concentrations of at-risk people. Further exploration of pop-up rapidtesting models is needed to better understand their specific role in facilitating high testing rates among at-risk populations and in reaching individuals who are long-term non-testers. In addition, the costeffectiveness of different testing models in NSW remains to be determined. New approaches to add to the current mix of testing options are anticipated and include innovations like self-testing and self-sample collection.

NSW Health and its partners are committed to escalating HIV testing, acknowledging that the elimination of HIV transmission will not be possible without it. Achieving this escalation means listening 
to affected communities and the health profession, and rethinking traditional approaches to testing in this new era of HIV.

Acknowledgements: This work was completed while Marianne Gale was an employee of the NSW Public Health Officer Training Program, funded by NSW Health.

Competing interests: No relevant disclosures.

Provenance: Not commissioned; not externally peer reviewed.

1 NSW Health, Communicable Diseases Branch. New diagnoses of HIV infection, NSW, 2012. http://www.health.nsw.gov.au/Infectious/hiv/ Documents/2012-hiv-summary.pdf (accessed Aug 2014).

2 NSW Health. NSW HIV Strategy 2012-2015. 2013 Annual Data Report. http://www.health.nsw.gov.au/endinghiv/Documents/ AnnualReportNSWHIVStrategy2012-2015DataReportFINAL.pdf (accessed Jul 2014).

3 Cohen MS, Chen YQ, McCauley M, et al. Prevention of HIV-1 infection with early antiretroviral therapy. N Engl J Med 2011; 365: 493-505.

4 Cohen J. Breakthrough of the year. HIV treatment as prevention. Science 2011; 334: 1628

5 Rodger A, Bruun T, Cambiano V, et al. HIV transmission risk through condomless sex if HIV+ partner on suppressive ART: PARTNER study. 21st Conference on Retroviruses and Opportunistic Infections; 2014 Mar 3-6; Boston, Mass, USA; abstract 153LB.
6 NSW Health. NSW HIV Strategy 2012-2015: a new era. Sydney: NSW Ministry of Health, 2012. http://www.health.nsw.gov.au/publications/ Publications/nsw-hiv-strategy-2012-15.pdf (accessed Jul 2014).

7 United Nations General Assembly. Political declaration on HIV and AIDS: intensifying our efforts to eliminate HIV and AIDS. Resolution 65/277. 10 Jun 2011. http://www.unaids.org/en/media/unaids/contentassets/ documents/document/2011/06/20110610_un_a-res-65-277_en.pdf (accessed Jul 2014).

8 Chang LW, Serwadda D, Quinn TC, et al. Combination implementation for HIV prevention: moving from clinical trial evidence to population-level effects. Lancet Infect Dis 2013; 13: 65-76.

9 Wilson DP, Hoare A, Regan DG, Law MG. Importance of promoting HIV testing for preventing secondary transmissions: modeling the Australian HIV epidemic among men who have sex with men. Sex Health 2009; 6: 19-33.

10 Sexually Transmissible Infections in Gay Men Action Group. Australian sexually transmitted infection and HIV testing guidelines 2014. STIGMA, 2014. http://stipu.nsw.gov.au/wp-content/uploads/STIGMA_Testing_ Guidelines_Final_v5.pdf (accessed Jul 2014).

11 Gray RT, Prestage GP, Down I, et al. Increased HIV testing will modestly reduce HIV incidence among gay men in NSW and would be acceptable if HIV testing becomes convenient. PLOS One 2013; 8: e55449.

12 NSW Health. HIV Support Program. http://www.health.nsw.gov.au/ Infectious/hiv/Pages/default.aspx (accessed Apr 2014). 